\title{
Convenient synthesis of phthalocyanines with disilazanes under mild conditions
}

\author{
Hitoshi Uchida, Masanari Mitsui, Paidi Yella Reddy, \\ Shuichi Nakamura, and Takeshi Toru* \\ Department of Applied Chemistry, Graduate School of Engineering, Nagoya Institute of \\ Technology, Gokiso, Showa-ku, Nagoya 466-8555, Japan; TEL and FAX: +81(52) 735-5217 \\ E-mail: toru@nitech.ac.jp
}

\section{Dedicated to Professor S. Swaminathan on his $80^{\text {th }}$ birthday}

(received 27 Aug 04; accepted 08 Oct 04; published on the web 27 Oct 04)

\begin{abstract}
We have previously reported convenient synthesis of metallo-and metal-free phthalocyanines under mild conditions by heating phthalimides, phthalic anhydrides, and phthalonitriles with hexamethyldisilazane. This paper deals with a convenient method for the preparation of $\mathrm{Cu}-$ and Zn-phthalocyanines as well as metal-free phthalocyanines by using disilazane derivatives with high boiling points.
\end{abstract}

Keywords: Metallophthalocyanine, metal-free phthalocyanine, hexamethyldisilazane, tetramethyldiphenyldisilazane, dimethyltetraphenyldisilazane

\section{Introduction}

A great deal of attention has been paid to optical and electronic properties of chemically and thermally stable phthalocyanines for developing new functionalized materials, ${ }^{1}$ e.g. optical information recording media, organic semiconductors for laser beam printers, ${ }^{2}$ agents for photodynamic therapy, ${ }^{3} \mathrm{NO}_{\mathrm{X}}{ }^{4,5}$ and halogen ${ }^{5}$ gas and $\mathrm{pH}$ sensors. ${ }^{6}$ Generally, metallophthalocyanines $^{7}$ as well as metal-free phthalocyanines ${ }^{8}$ have been synthesized from phthalonitrile derivatives under basic conditions. Urea is used in the production of phthalocyanines from less expensive starting materials such as phthalic acids, phthalic anhydrides and phthalimides but the reaction needs severe conditions which often cause the formation of undesirable by-products. ${ }^{9}$

Recently, we have reported that hexamethyldisilazane ${ }^{10}$ (HMDS) is an excellent reagent for the construction of the phthalocyanine framework from phthalimides, phthalic anhydrides, and phthalonitriles. ${ }^{11}$ Various metallophthalocyanines can be prepared under mild conditions on 
heating with HMDS. HMDS also realized the first synthesis of metal-free phthalocyanines from phthalimides and phthalic anhydrides. HMDS is a nitrogen source and it also accelerates dehydration in the formation of the phthalocyanine framework. The reaction using phthalimides or phthalic anhydrides as starting materials can be efficiently performed at $150{ }^{\circ} \mathrm{C}$; however, the reaction should be carried out in a sealed vessel because of the low boiling point of HMDS. We now report a successful use of disilazane derivatives having high boiling points in place of HMDS in the preparation of phthalocyanines on heating without using a sealed vessel.

\section{Results and Discussion}

The reaction of phthalimides with various disilazane derivatives was examined. The results are shown in Table 1. As previously reported, ${ }^{11 \mathrm{~b}}$ HMDS 1a gives Cu-phthalocyanine $\mathbf{C u}-\mathbf{2}$ in 71\% yield by heating phthalimide with 0.25 equiv of $\mathrm{Cu}(\mathrm{OTf})_{2}$ and $\mathrm{DMF}$ at $150{ }^{\circ} \mathrm{C}$ for $10 \mathrm{~h}$ (entry 1 ). The boiling point of HMDS is $126^{\circ} \mathrm{C}$ and bis(trimethylsilyl) ether, which is one of the products formed during the reaction, has even lower boiling point. The reaction should be performed in a sealed vessel to maintain the reaction temperature at $150{ }^{\circ} \mathrm{C}$. Thus, the reaction with disilazanes having higher boiling points was examined. The reaction was carried out as in the case using HMDS except that it was done under atmospheric pressure. A mixture of phthalimide, disilazane, $\mathrm{Cu}(\mathrm{OTf})_{2}$, and DMF in a molar ratio of 1.0:4.0:0.25:1.0 was heated at $150{ }^{\circ} \mathrm{C}$ for $10 \mathrm{~h}$. The 8and 5-membered cyclic derivatives $\mathbf{1 b}$ and $\mathbf{1 c}$ afforded $\mathbf{C u}-\mathbf{2}$ in 35 and $42 \%$ yields, respectively (entries 2 and 3). The boiling point of the corresponding disilyl ethers, which are supposed to be one of the products formed during the formation of the Pc framework, are also shown for reference in Table 1. Both 1,1,3,3-tetramethyl-1,3-diphenyldisilazane 1d and 1,3-dimethyl1,1,3,3-diphenyldisilazane 1e having high boiling points showed high yields of $\mathbf{C u}-2$ after heating at $150{ }^{\circ} \mathrm{C}$ for $10 \mathrm{~h}$ under atmospheric pressure (entries 4 and 5). Since disilyl ethers corresponding to $1 \mathbf{d}$ and $1 \mathrm{e}$ also have boiling points higher than $150{ }^{\circ} \mathrm{C}$, the reaction temperature could be maintained at $150{ }^{\circ} \mathrm{C}$.

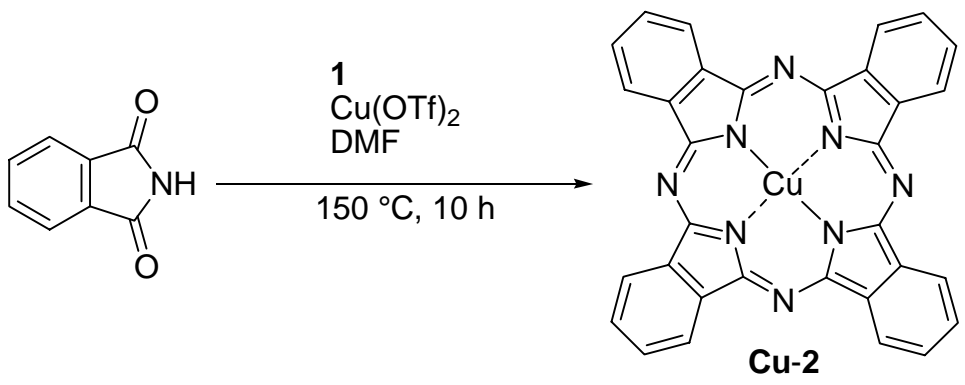


Table 1. Formation of Cu-phthalocyanine $\mathbf{C u}-\mathbf{2}$ on treatment of phthalimide with various disilazanes, $\mathrm{Cu}(\mathrm{OTf})_{2}$, and $\mathrm{DMF}$

\begin{tabular}{|c|c|c|c|c|c|}
\hline Entry & Disilazane & bp & $\begin{array}{l}\text { Reaction } \\
\text { Apparatus }^{\text {a }}\end{array}$ & $\begin{array}{l}\text { Yield of } \\
\text { Cu-2 } \\
(\%)\end{array}$ & $\begin{array}{l}\text { Disilyl Ethers } \\
\text { bp }\end{array}$ \\
\hline 1 & $\begin{array}{l}\mathrm{Me}_{3} \mathrm{Si}_{\substack{N^{-} \\
\mathrm{HiMe}_{3}}}{ }^{\mathrm{H}} \\
\mathbf{1 a}\end{array}$ & $126^{\circ} \mathrm{C}$ & A & $71^{\mathrm{b}}$ & $\begin{array}{l}\mathrm{Me}_{3} \mathrm{Si}{ }_{-}-\mathrm{SiMe}_{3} \\
99{ }^{\circ} \mathrm{C}\end{array}$ \\
\hline 2 & 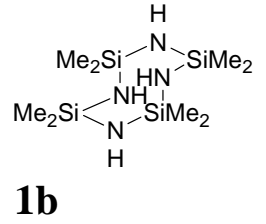 & $225^{\circ} \mathrm{C}$ & B & 35 & \\
\hline 3 & $\begin{array}{l}\mathrm{Me}_{2} \mathrm{Si}_{{ }_{N}{ }^{-} \text {SiMe }_{2}} \\
\mathbf{H}\end{array}$ & $\begin{array}{l}92^{\circ} \mathrm{C} / \\
185 \\
\mathrm{mmHg}\end{array}$ & B & 42 & $\begin{array}{l}\mathrm{Me}_{2} \mathrm{Si}_{-} \mathrm{O}^{-\mathrm{SiMe}_{2}} \\
124{ }^{\circ} \mathrm{C}\end{array}$ \\
\hline 4 & $\begin{array}{l}\mathrm{PhMe}_{2} \mathrm{Si}_{-\mathrm{N}^{-}}-\mathrm{SiMe}_{2} \mathrm{Ph} \\
\mathbf{H} \\
\mathbf{1 d}\end{array}$ & $\begin{array}{l}96^{\circ} \mathrm{C} / \\
0.1 \mathrm{mmHg}\end{array}$ & B & 76 & $\begin{array}{l}\mathrm{PhMe}_{2} \mathrm{Si}_{-\mathrm{O}_{-}-\mathrm{SiMe}_{2} \mathrm{Ph}} \\
96^{\circ} \mathrm{C} / \\
0.1 \mathrm{mmHg}\end{array}$ \\
\hline 5 & $\begin{array}{l}\mathrm{Ph}_{2} \mathrm{MeSi}_{-{ }^{-}}-\mathrm{SiMePh}_{2} \\
\mathbf{1 e}\end{array}$ & $\begin{array}{l}218^{\circ} \mathrm{C} / \\
1.5 \mathrm{mmHg}\end{array}$ & B & 75 & $\begin{array}{l}\mathrm{Ph}_{2} \mathrm{MeSi}_{>} \mathrm{O}_{-} \mathrm{SiMePh}_{2} \\
155^{\circ} \mathrm{C} / \\
13 \mathrm{mmHg}\end{array}$ \\
\hline
\end{tabular}

${ }^{\mathrm{a}}$ The reaction was performed at $150{ }^{\circ} \mathrm{C}$ for $10 \mathrm{~h}$ in a sealed vessel (A), or under atmospheric pressure (B).

${ }^{\mathrm{b}}$ Ref. $11 \mathrm{~b}$.

The preparation of various phthalocyanines was next examined using the disilazane 1d (Table 2). Zn-Phthalocyanine Zn-2 was obtained in the reaction of phthalimide with Zn(OTf) 2 (entry 1). Cu-Phthalocyanines were prepared by heating various phthalimides with disilazane 1d and $\mathrm{Cu}(\mathrm{OTf})_{2}$ under the reaction conditions similar to the above. Thus, Cu-phthalocyanines $\mathbf{C u}-$ 3-6 having a variety of substituents were produced in good yields from 4-tert-butyl, 4-methyl, 4,5-dichloro-, and 3,4,5,6-tetrachlorophthalimides (entries 2-5). Phthalocyanine derivatives Cu-3 and $\mathbf{C u}-\mathbf{4}$ were obtained as a mixture of regioisomers which were not separated. The reaction of phthalimides with 1d in the absence of a metal salt gave $\mathbf{H}_{2}-\mathbf{2}$ in $40 \%$ yield (entry 6). Addition of $\left(\mathrm{NH}_{4}\right)_{2} \mathrm{SO}_{4}$ improved the yield (entry 7). The role of $\left(\mathrm{NH}_{4}\right)_{2} \mathrm{SO}_{4}$ is not clear, but it would activate the disilazane and/or the phthalimide to promote the formation of the phthalocyanine framework. When $\mathrm{Cu}(\mathrm{OTf})_{2}$ and $\mathrm{Zn}(\mathrm{OTf})_{2}$ are used, it is not necessary to use $\left(\mathrm{NH}_{4}\right)_{2} \mathrm{SO}_{4}$ because these triflates and/or TfOH liberated during the reaction would activate the disilazane and phthalimide. ${ }^{11 b, c}$ Peripherally substituted metal-free phthalocyanines $\mathbf{H}_{\mathbf{2}}-\mathbf{3}$ and $\mathbf{H}_{\mathbf{2}} \mathbf{- 4}$ could also be prepared (entries 8 and 9). 


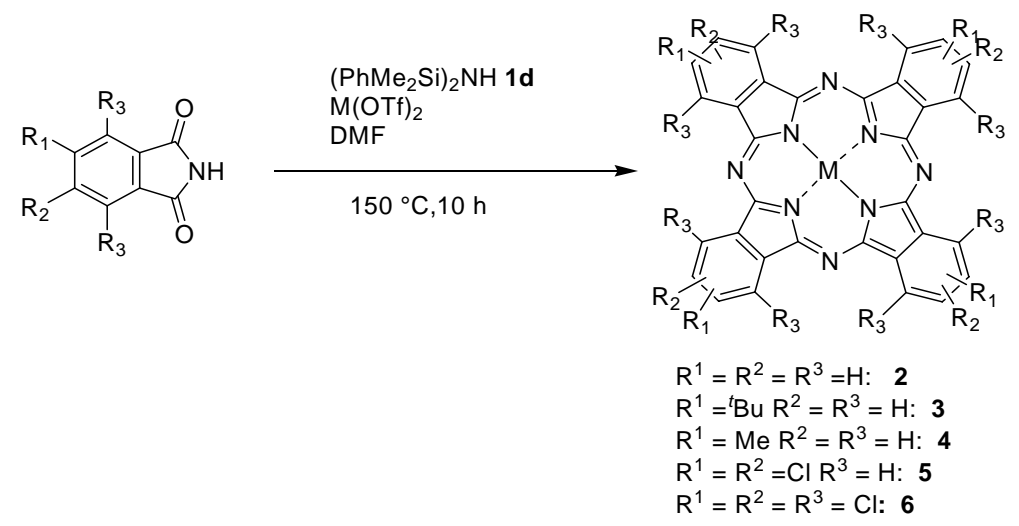

Table 2. Reaction of $\mathbf{1}$ with disilazane $\mathbf{1 d}$

\begin{tabular}{|c|c|c|c|c|c|c|c|}
\hline \multirow[t]{2}{*}{ Entry } & \multicolumn{3}{|c|}{ Phthalimide } & \multirow[t]{2}{*}{$\mathrm{M}(\mathrm{OTf})_{2}$} & \multirow[t]{2}{*}{ Additive } & \multirow[t]{2}{*}{ Product } & \multirow{2}{*}{$\begin{array}{l}\text { Yield } \\
\text { (\%) }\end{array}$} \\
\hline & $\mathrm{R}_{1}$ & $\mathrm{R}_{2}$ & $\mathrm{R}_{3}$ & & & & \\
\hline 1 & $\mathrm{H}$ & $\mathrm{H}$ & $\mathrm{H}$ & $\mathrm{Zu}(\mathrm{OTf})_{2}$ & - & $\mathrm{Zu}-\mathbf{2}$ & 38 \\
\hline 2 & $\mathrm{t}-\mathrm{Bu}$ & $\mathrm{H}$ & $\mathrm{H}$ & $\mathrm{Cu}(\mathrm{OTf})_{2}$ & - & Cu-3 & 81 \\
\hline 3 & $\mathrm{Me}$ & $\mathrm{H}$ & $\mathrm{H}$ & $\mathrm{Cu}(\mathrm{OTf})_{2}$ & - & $\mathrm{Cu}-4$ & 65 \\
\hline 4 & $\mathrm{Cl}$ & $\mathrm{Cl}$ & $\mathrm{H}$ & $\mathrm{Cu}(\mathrm{OTf})_{2}$ & - & $\mathrm{Cu}-5$ & 27 \\
\hline 5 & $\mathrm{Cl}$ & $\mathrm{Cl}$ & $\mathrm{Cl}$ & $\mathrm{Cu}(\mathrm{OTf})_{2}$ & - & $\mathrm{Cu}-6$ & 11 \\
\hline 6 & $\mathrm{H}$ & $\mathrm{H}$ & $\mathrm{H}$ & - & - & $\mathbf{H}_{2}-2$ & 40 \\
\hline 7 & $\mathrm{H}$ & $\mathrm{H}$ & $\mathrm{H}$ & - & $\left(\mathrm{NH}_{4}\right)_{2} \mathrm{SO}_{4}$ & $\mathbf{H}_{2}-2$ & 61 \\
\hline 8 & $\mathrm{t}-\mathrm{Bu}$ & $\mathrm{H}$ & $\mathrm{H}$ & - & $\left(\mathrm{NH}_{4}\right)_{2} \mathrm{SO}_{4}$ & $\mathbf{H}_{2}-3$ & 15 \\
\hline 9 & $\mathrm{Me}$ & $\mathrm{H}$ & $\mathrm{H}$ & - & $\left(\mathrm{NH}_{4}\right)_{2} \mathrm{SO}_{4}$ & $\mathrm{H}_{2}-4$ & 59 \\
\hline
\end{tabular}

In summary, unsubstituted or peripherally substituted $\mathrm{Cu}$ - and $\mathrm{Zn}$-phthalocyanines and metal-free phthalocyanines were conveniently prepared from phthalimides on treatment with disilazane derivatives having high boiling points.

\section{Experimental Section}

Preparation of Cu-phthalocyanine $\mathrm{Cu}$-2 with 1,1,3,3-tetramethyl-1,3-diphenyldisilazane. Phthalimide (100 mg, $0.68 \mathrm{mmol}$ ), copper(II) triflate (69 mg, $0.17 \mathrm{mmol}$ ), 1,1,3,3-tetramethyl1,3-diphenyldisilazane (790 $\mu \mathrm{L}, 777 \mathrm{mg}, 2.7 \mathrm{mmol}$ ), and DMF (50 $\mu \mathrm{L}, 0.68 \mathrm{mmol}$ ) were mixed in a round-bottom flask equipped with a reflux condenser. The mixture was heated at $150{ }^{\circ} \mathrm{C}$ under argon. A dark blue solid immediately appeared. After being heated for $10 \mathrm{~h}$, the mixture was cooled and filtered. The solid was washed with methanol and then dissolved in concentrated $\mathrm{H}_{2} \mathrm{SO}_{4}(5 \mathrm{~mL})$. The solution was poured into water $(100 \mathrm{~mL})$. The resulting blue precipitates were filtered and washed successively with dilute $\mathrm{H}_{2} \mathrm{SO}_{4}$, water, and methanol. The solid was 
further purified by extraction with methanol by Soxhlet's extractor to give $75 \mathrm{mg}$ (76\%) of $\mathbf{C u}-\mathbf{2}$ as a blue solid.

Preparation of $\mathrm{Cu}$-phthalocyanine $\mathrm{Cu}-2$ with 1,3-dimethyl-1,1,3,3-tetraphenyldisilazane. A mixture of phthalimide (100 mg, $0.68 \mathrm{mmol}$ ), copper(II) triflate (69 mg, $0.17 \mathrm{mmol}), 1,3$ dimethyl-1,1,3,3-tetraphenyldisilazane (1.0 g, $2.7 \mathrm{mmol}$ ), and DMF (50 $\mu \mathrm{L}, 0.68 \mathrm{mmol}$ ) was heated at $150{ }^{\circ} \mathrm{C}$ for $10 \mathrm{~h}$. Workup and purification as above gave $74 \mathrm{mg}$ (75\%) of $\mathbf{C u}-2$ as a blue solid.

Preparation of Cu-phthalocyanine Cu-2 with 2,2,5,5-tetramethyl-2,5-disila-1azacyclopentane. A mixture of phthalimide (100 mg, $0.68 \mathrm{mmol}$ ), copper(II) triflate (69 mg, $0.17 \mathrm{mmol}), 2$,2,5,5-tetramethyl-2,5-disila-1-azacyclopentane (1.0 mL, $867 \mathrm{mg}, 2.7 \mathrm{mmol})$, and DMF (50 $\mu \mathrm{L}, 0.68 \mathrm{mmol}$ ) was heated at $150{ }^{\circ} \mathrm{C}$ for $10 \mathrm{~h}$. Workup and purification as above gave $41 \mathrm{mg}$ (42\%) of $\mathbf{C u}-2$ as a blue solid.

Preparation of $\mathrm{Cu}$-phthalocyanine $\mathrm{Cu}-2$ with 2,2,4,4,6,6,8,8-octamethylcyclotetrasilazane. A mixture of phthalimide (100 mg, $0.68 \mathrm{mmol}$ ), copper(II) triflate (69 $\mathrm{mg}, 0.17 \mathrm{mmol}$ ), 1,1,3,3,5,5,7,7-octamethylcyclotetrasilazane (795 mg, $2.7 \mathrm{mmol}$ ), and DMF (50 $\mu \mathrm{L}, 0.68 \mathrm{mmol}$ ) was heated at $150{ }^{\circ} \mathrm{C}$ for $10 \mathrm{~h}$ to giv $34 \mathrm{mg}$ (35\%) of $\mathbf{C u}-2$ as a blue solid.

Preparation of Zn-phthalocyanine Zn-2 with 1,1,3,3-tetramethyl-1,3-diphenyldisilazane. A mixture of phthalimide (100 mg, $0.68 \mathrm{mmol}$ ), zinc triflate (69 mg, $0.17 \mathrm{mmol}), 1,1,3,3-$ tetramethyl-1,3-diphenyldisilazane (790 $\mu \mathrm{L}, 777 \mathrm{mg}, 2.7 \mathrm{mmol}$ ), and DMF (50 $\mu \mathrm{L}, 0.68 \mathrm{mmol}$ ) was heated at $150{ }^{\circ} \mathrm{C}$ for $10 \mathrm{~h}$ to give $37 \mathrm{mg}$ (38\%) of $\mathbf{Z n - 2}$ as a blue solid.

Preparation of tetra-tert-butyl-Cu-phthalocyanine Cu-3. A mixture of 4-tertbuthylphthalimide (140 mg, $0.69 \mathrm{mmol}$ ), copper(II) triflate (69 mg, $0.17 \mathrm{mmol}), 1,1,3,3-$ tetramethyl-1,3-diphenyldisilazane (800 $\mu \mathrm{L}, 787 \mathrm{mg}, 2.8 \mathrm{mmol}$ ), and DMF (50 $\mu \mathrm{L}, 0.68 \mathrm{mmol}$ ) was heated at $150{ }^{\circ} \mathrm{C}$ for $10 \mathrm{~h}$. A dark purple solid immediately appeared. After being heated for $10 \mathrm{~h}$, the mixture was cooled and filtered. The solid was washed with methanol and then dissolved in concentrated $\mathrm{H}_{2} \mathrm{SO}_{4}(5 \mathrm{~mL})$. The solution was poured into water $(100 \mathrm{~mL})$. The resulting blue precipitates were filtered and washed successively with dilute $\mathrm{H}_{2} \mathrm{SO}_{4}$, water, and methanol. The solid was further purified by silica gel column chromatography (benzene/THF=99/1) to give $112 \mathrm{mg}(81 \%)$ of $\mathbf{C u}-3$ as a blue solid.

Preparation of tetramethyl-Cu-phthalocyanine Cu-4. A mixture of 4-methylphthalimide (110 mg, $0.68 \mathrm{mmol}$ ), copper(II) triflate (69 mg, $0.17 \mathrm{mmol}$ ), 1,1,3,3-tetramethyl-1,3diphenyldisilazane (790 $\mu \mathrm{L}, 787 \mathrm{mg}, 2.8 \mathrm{mmol}$ ), and DMF (50 $\mu \mathrm{L}, 0.68 \mathrm{mmol})$ was heated at $150{ }^{\circ} \mathrm{C}$ for $10 \mathrm{~h}$. Workup and purification as shown in the preparation of $\mathrm{Cu}-2$ with 1,1,3,3tetramethyl-1,3-diphenyldisilazane gave $70 \mathrm{mg}$ (65\%) of $\mathbf{C u}-\mathbf{4}$ as a blue solid.

Preparation of octachloro-Cu-phthalocyanine Cu-5. A mixture of 4,5-dichlorophthalimide (145 mg, $0.67 \mathrm{mmol}$ ), copper(II) triflate (68 mg, $0.17 \mathrm{mmol}$ ), 1,1,3,3-tetramethyl-1,3diphenyldisilazane (780 $\mu \mathrm{L}, 767 \mathrm{mg}, 2.7 \mathrm{mmol}$ ), and DMF (50 $\mu \mathrm{L}, 0.68 \mathrm{mmol}$ ) was heated at $150{ }^{\circ} \mathrm{C}$ for $10 \mathrm{~h}$. Usual workup and purification gave $39 \mathrm{mg}$ (27\%) of $\mathbf{C u}-\mathbf{5}$ as a blue solid.

Preparation of hexadecachloro-Cu-phthalocyanine Cu-6. A mixture of 3,4,5,6tetrachlorophthalimide (195 mg, $0.68 \mathrm{mmol}$ ), copper(II) triflate (69 mg, $0.17 \mathrm{mmol}$ ), 1,1,3,3- 
tetramethyl-1,3-diphenyldisilazane (790 $\mu \mathrm{L}, 777 \mathrm{mg}, 2.7 \mathrm{mmol}$ ), and DMF (50 $\mu \mathrm{L}, 0.68 \mathrm{mmol}$ ) was heated at $150{ }^{\circ} \mathrm{C}$ for $10 \mathrm{~h}$. Usual workup and purification gave $21 \mathrm{mg}$ (11\%) of Cu-6 as a blue solid.

Preparation of $\mathbf{H}_{2}$-phthalocyanine $\mathbf{H}_{2}$-2. (A) A mixture of phthalimide (100 $\mathrm{mg}, 0.68 \mathrm{mmol}$ ), 1,1,3,3-tetramethyl-1,3-diphenyldisilazane (790 $\mu \mathrm{L}, 777 \mathrm{mg}, 2.7 \mathrm{mmol}$ ), and DMF (50 $\mu \mathrm{L}$, $0.68 \mathrm{mmol}$ ) was heated at $150{ }^{\circ} \mathrm{C}$ for $10 \mathrm{~h}$ to give $39 \mathrm{mg}(40 \%)$ of $\mathbf{H}_{2}$-2 as a blue solid. (B) The same procedure as in A except the addition of ammonium sulfate ( $9 \mathrm{mg}, 0.07 \mathrm{mmol}$ ) gave $59 \mathrm{mg}$ (61\%) of $\mathbf{H}_{2}$-2.

Preparation of tetra-tert-butyl-H $\mathbf{H}_{2}$-phthalocyanine $\mathbf{H}_{2}$-3. A mixture of 4-tertbuthylphthalimide (140 mg, $0.69 \mathrm{mmol}$ ), ammonium sulfate (9 mg, $0.07 \mathrm{mmol}), 1,1,3,3-$ tetramethyl-1,3-diphenyldisilazane (800 $\mu \mathrm{L}, 787 \mathrm{mg}, 2.8 \mathrm{mmol}$ ), and DMF (50 $\mu \mathrm{L}, 0.68 \mathrm{mmol}$ ) was added. Then, the mixture was heated at $150{ }^{\circ} \mathrm{C}$ for $10 \mathrm{~h}$. Workup and purification by silica gel column chromatography as above gave $19 \mathrm{mg}$ (15\%) of $\mathbf{H}_{2}-\mathbf{3}$ as a blue solid.

Preparation of tetramethyl- $\mathbf{H}_{2}$-phthalocyanine $\mathbf{H}_{2}-4$. A mixture of 4-methylphthalimide (110 mg, $0.68 \mathrm{mmol}$ ), ammonium sulfate (9 mg, $0.07 \mathrm{mmol}), 1,1,3,3$-tetramethyl-1,3diphenyldisilazane (790 $\mu \mathrm{L}, 787 \mathrm{mg}, 2.8 \mathrm{mmol}$ ), and DMF (50 $\mu \mathrm{L}, 0.68 \mathrm{mmol}$ ) was heated at $150{ }^{\circ} \mathrm{C}$ for $10 \mathrm{~h}$. Usual workup and purification as above gave $57 \mathrm{mg}$ (59\%) of $\mathbf{H}_{\mathbf{2}} \mathbf{- 4}$ as a blue solid.

\section{Acknowledgements}

This work was partly supported by a Grant- in- Aid for Scientific Research on Priority Areas (A) "Exploitation of Multi- Element Cyclic Molecules" (No. 14044040) from the Ministry of Education, Culture, Sports, Science, and Technology of Japanese Government.

\section{References}

1. (a) Moser, F. H. The Phthalocyanines: Properties, Vols. 1 and 2; Thomas, A. L., Ed.; CRC: Boca Raton, 1983. (b) Phthalocyanines: Properties and Applications, Leznoff, C. C.; Lever, A. B. P., Eds,; VCH: New York, Vol. 1, 1989; Vols. 2 and 3, 1993; Vol. 4; 1996. (c) Thomas, A. L. The Phthalocyanines: Applications; CRC: Boston, 1990. (d) McKeown, N. B. Phthalocyanine Materials: Synthesis, Structure and Function; Cambridge University Press: Cambridge, 1998. (e) Hanack, M.; Heckmann, H.; Polley, R. Methods of Organic Chemistry, Vol. E9d; Schaumann, E., Ed.; Thieme: Stuttgart, 1998, pp 717-846. (f) G. Torre; C. G. Claessesns; T. Torres, Eur. J. Org. Chem. 2000, 2821.

2. Arishima, K.; Hiratsuka, H.; Tate, A.; Okada, T. Appl. Phys. Lett. 1982, 40, 279.

3. (a) Wagner, J. R.; Ali, H.; Langlois, E.; Brasseuseur, N.; van Lier, J. E. Photochem. Photobiol. 1987, 45, 587. (b) Brasseur, N.; Langlois, E.; Ali, H.; van Lier, J. E. Photochem. Photobiol. 
1987, 46, 739. (c) Paquette, B.; Ali, H.; Langlois, R.; van Lier, J. E. Photochem. Photobiol. 1988, 47, 215. (d) Brasseur, N.; Ali, H.; Langlois, E.; van Lier, J. E. Photochem. Photobiol. 1988, 47, 705. (e) Ali, H.; Langlois, E.; Wagner, J. R.; Brasseur, N.; Paquette, B.; van Lier, J. E. Photochem. Photobiol. 1988, 47, 713.

4. Bott, B.; Jones, T. A. Sens. Actuators 1984, 5, 43.

5. Jones, T. A.; Bott, B. Sens. Actuators 1986, 9, 27.

6. Sauer, T.; Caseri, W.; Wegner, G.; Vogel, A.; Hoffmann, B. J. Phys. D: Appl. Phys. 1990, 23, 79.

7. (a) Barrett, P. A.; Frye, D. A.; Linstead, R. P. J. Chem. Soc. 1918, 1157. (b) Oksengendler, I. G.; Kondratenko, N. V.; Luk’yanets, E. A. Zh. Obshch. Khim. 1978, 14, 1046; J. Org. Chem. USSR 1978, 14, 976. (c) Wöhrle, D.; Meyer, G.; Wahl, B. Makromol. Chem. 1980, 181, 2127. (d) McKeown, N. B.; Chambrier, I.; Cook, M, J. J. Chem. Soc., Perkin Trans. 1 1990, 1169. (e) Guyon, F.; Pondaven, A.; Guenot, P.; L’Her, M. Inorg. Chem. 1994, 33, 4787. (f) Cook, M. J.; Hersans, R.; McMurdo, J.; Russell, D. A. J. Mater. Chem. 1996, 6, 149. (g) Terekhov, D. S.; Nolan, K. L. M.; McAuthur, C. R.; Leznoff, C. C. J. Org. Chem. 1996, 61, 3034. (h) Kobayashi, N.; Higashi, R.; Ishii, K.; Hatsusaka, K.; Ohta, K. Bull. Chem. Soc. Jpn. 1999, 72, 1263. (i) Lenzoff, C. C.; D’ascanio, A. M.; Yildiz, S. Z.; J. Porphyrins Phthalocyanines 2000, 4, 103.

8. (a) Tomoda, H.; Saito, S.; Ogawa, S.; Shiraishi, S. Chem. Lett. 1980, 1277. (b) Tomoda, H.; Saito, S.; Shiraishi, S. Chem. Lett. 1981, 313. (c) Edmondson S. J.; Hill, J. S.; Mitchell, P. C. H. J. Chem. Soc. Dalton Trans. 1990, 1115. (d) Wöhrle, D.; Schnurpfeil, G.; Knothe, G. Dyes Pigm. 1992, 18, 91. (e) Linssen, T. G.; Dürr, K.; Hanack, M.; Hirsch, A. J. Chem. Soc., Chem. Commun. 1995, 103.

9. Yao, J.; Yonehara, H.; Pac, C. Bull. Chem. Soc. Jpn. 1995, 68, 1001.

10. (a)Vorbrüggen, H. Acc. Chem. Res. 1995, 28, 509. (b) Vorbrüggen, H.; Krolicz, K. Liebigs Ann. Chem. 1976, 745.

11. (a) Uchida H.; Tanaka H.; Yoshiyama H.; Reddy P. Y.; Nakamura S.; Toru T. Synlett 2002, 1649. (b) Uchida H.; Reddy P. Y.; Nakamura S.; Toru T. J. Org. Chem. 2003, 68, 8736. (c) Uchida H.; Yoshiyama H.; Reddy P. Y.; Nakamura S.; Toru T. Synlett 2003, 2083. (d) Uchida H.; Yoshiyama H.; Reddy P. Y.; Nakamura S.; Toru T. Bull. Chem. Soc. Jpn. 2004, 77, 1401. 